\section{Epigenetic Regulation of Allergic Disease}

The hygiene hypothesis is currently the leading concept to explain the dramatic increase in chronic inflammatory disease including autoimmunities and allergies. Conversely, several gram-positive and gram-negative bacteria have been isolated from rural environmental communities which provide protection of allergic asthma. Overwhelming evidence indicates a strong impact of environmental microbes on the programming and the development of (early) immune responses. Based on clinical and epidemiological data, a certain exposure of environmental microbes - particularly of bacteria - seems to be an important pre-requisite in order to program immune responses towards the tolerance default program. This prevents unwanted (chronic) inflammatory diseases, such as allergies and autoimmune diseases of the gut, the brain, or other organs. Microbes facilitate the immunoregulatory effects via epigenetic mechanisms. The Grand challenge is to define the appropriate microbial environment on the cellular and molecular level in order to delineate the underlying mechanism of microbehost interaction. An important concept in this context is microbial diversity. Reduced diversity is closely linked to several clinical phenotypes, such as allergies and asthma and precedes the clinical onset of the disease, suggesting a cause-effect relationship. This concept implies the loss of (ancient) evolutionary co-evolved microbial strains and is the result of changes in lifestyle condition, particularly under westernized and industrialized environmental conditions. The great challenge in this research field is to delineate the molecular pathomechanism of gene-environment interactions and the impact of microbial communities on this complex and intimate relationship. Epigenetic mechanisms link on the functional level the interaction between (environmental) microbes and immunological programming. Only through better understanding of these mechanisms, we will be able to define novel and attractive strategies for the prevention of chronic inflammatory diseases. Therefore, it is urgently needed to move this research field towards translational activities. The next few years will provide a compelling amount of novel data, which will hopefully improve the understanding of mechanisms of this important communication between the host and the microbial communities.

\section{Conflict of Interest}

Research support: DFG, BMBF, EU, Land Hessen, DAAD, ALK, Stiftung Pathobiochemie, Ernst-Wendt-Stiftung, Mead Johnson Nutritional, Beckman Coulter. Speakers honorarium: Allergopharma, Novartis, ThermoFisher, Danone, Mead Johnson Nutritional, Bencard. Consulting: Bencard, sterna-biologicals (cofounder).

\section{Author}

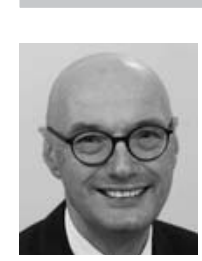

\section{Harald Renz}

MD, Institute of Laboratory Medicine, Philipps University Marburg, Germany

\section{Correspondence}

\section{Harald Renz, MD}

Institute of Laboratory Medicine Philipps University Marburg, Germany Baldingerstraße 35043 Marburg Germany

harald.renz@uk-gm.de 